\title{
Protective, preventive and enhancing-memory effects of the flavonoidic-rich plants or flavonoidic molecules: behavioural, neurochemical and pharmacological correlates
}

\begin{abstract}
Our group has focused on understanding the putative neuromodulatory effects of flavonoidic-rich plants or flavonoidic molecules. This mini-review summarizes the effects of these substances on fear memory and anxiety in adult and middle-aged rats Multipledosing schemes, different behavioral procedures and different time points of treatment have provided direct evidence of the modulatory effects of these molecules upon the acquisition and extinction of conditioned suppression. This has enabled us to further evaluate shortand long-term memory. To better understand the effects of standardized extract of Ginkgo biloba $(\mathrm{EGb})$ or of Flavonoidic fraction $(\mathrm{FfB})$ on fear memory, we conducted both pharmacological and molecular analysis. The dose-dependent effects observed after EGb or FfB treatments on the conditioned fear include the spontaneous recovery of fear memory. FfB reversed the effect of blocking of GluN2B subunits of $N$-methyl-D-Aspartate receptor (GluN2B-NMDARs) on conditioned suppression. These results turned our attention to the therapeutic relevance of these data, as the pharmacological agents that enhance the acquisition/consolidation of fear memory or fear extinction might serve as an adjunct to the treatment of memory decline or anxiety disorders. In addition, the molecular results provided important information regarding the role of the dorsal hippocampal formation (dHF) on conditioned suppression and as a possible target to potential drug therapy. Additionally, we evaluated the effects of EGb on short and long term-memory in middleaged rats submitted to the plus maze discriminative avoidance task (PM-DAT) as well as upon the level of DNA damage of Prefrontal cortex (PFC) and dHF. All together, these findings appear to support and extend the view that flavonoids are cognitive enhancers.
\end{abstract}

Volume 6 Issue 5 - 2017

\section{Claudia Raquel Zamberlam, Suzete Maria Cerutti \\ Department of Biological Science, Federal University of São} Paulo, Brazil

Correspondence: Suzete Maria Cerutti, PhD. Cellular and Behavioral Pharmacology Laboratory, Department of Biological Science, Universidade Federal de São Paulo, Brazil, Tel 55- I I3319-3300,Email smcerutti@unifesp.br

Received: April 07, 2017 | Published: April 27, 2017

Keywords: flavonoidic fraction b (FfB), ginkgo biloba, conditioned lick suppression, extinction fear memory, dorsal hippocampal formation, prefrontal cortex

\section{Introduction}

Converging evidence over the last few decades has shown that extracts of flavonoid-rich plant or flavonoid molecules have been the focus of research as modulators of brain function, neuroprotective, chemopreventive and anti-anxiety properties and memory-enhancing effects. ${ }^{1-6}$ It has been suggested that the ability to modulate the glutamatergic, serotoninergic, and GABAergic neurotransmission is attributable to flavonoid-induced effects. Additionally, their antioxidant activity, which is recognized by their ability to inhibit the production of free radicals, and their neuroprotective effects were found to be related. ${ }^{4}$ Although studies ex vivo, in vivo, and in vitro have provided evidence supporting the effects of flavonoids on the central nervous system, the cellular and molecular pathways through which these compounds modulate their effects are not completely understood. Our group has sought to elucidate the role of flavonoid-rich extract or flavonoidic fraction in fear memory and anxiety using a combination of molecular, behavioral and pharmacological analysis. Our findings regarding the cellular and molecular mechanisms of flavonoids action appear promising with respect to the development of new therapeutic strategies in the potential treatment or prevention of cognitive deficits or anxiety disorders.

Ginkgo bilobaimproves short and long-term fear memory by modulating dorsal hippocampal formation and prefrontal cortex

Studies in our laboratory have characterized the putative neuromodulatory effects of short and long-term treatment with flavonoid-rich plant extracts, from a standardized extract of the green leaves of Ginkgo biloba (EGb) ${ }^{7,8}$ on conditioned lick suppression (CLS) in adult healthy Wistar rats. These behavioral effects were associated with differential expression (mRNA and protein) of CAMP responsive element binding protein-1 (CREB-1), grown associated protein-43 (GAP-43) and glial fibrillary acidic protein (GFAP) in the dorsal hippocampus (DH), the prefrontal cortex and the amygdaloid complex. More recently we showed that EGb-treatment, in a dosedependent manner, modulates short- and long-term fear memory during its acquisition and extinction on CLS task, suggesting that different neurochemical systems can be involved in the acquisition and retrieval of fear memory. Further, the reduced fear expression during retrieval might be related to an anti-anxiety effect rather than to mnemonic effects, as previously hypothesized by us. ${ }^{6}$

Consistent with this evidence, in another published study, we showed that long-term treatment with EGb improved the short-term memory in middle-aged rats on plus-maze discriminative avoidance task (PM-DAT), which may be associated with reductions in free radical production in the prefrontal cortex. ${ }^{5}$ This effect appeared to be associated with reduced DNA damage in the prefrontal cortex of middle-aged animals that received long-term pre-treatment with $\mathrm{EGb}$ before and after cerebral H2O2-induced toxicity. It thus appears that treatment with EGb protects prefrontal cortex from damage and enhances the short-term memory.

Together, our data provides important information concerning the effects of EGb and suggests that EGb may represent a promising drug 
to help prevent or treat neurocognitive impairments and prevent the decline of memory due to aging. Also, our data suggested that both the $\mathrm{PFC}$ and $\mathrm{dHF}$ are involved in modulatory effects of EGb on short- and long-term fear memory.

Similar results were seen after a single dose treatment with crude extract of Erythrina falcata (CE), pure flavonoids or flavonoid-rich fractions from the stem bark of E. falcata using a one-trial, step-down inhibitory avoidance (IA) ${ }^{9}$ or conditioned lick suppression (CLS) ${ }^{8}$ tasks.

\section{Flavonoidic fraction improves the acquisition of fear memory without preventing its extinction: role of $\mathrm{dHF}$}

Our data has established that the treatment with flavonoid-rich plants or flavonoidic molecules resulted in a significant retention of long-term fear memory and its spontaneous recovery evaluated during extinction test sessions, although FfB did not prevent the acquisition of the extinction of fear conditioning within-sessions. ${ }^{6,9}$ Moreover, our data showed, through pharmacological manipulation and genetic analysis, that acquisition of lick suppression is mediated by hydroxytryptamine (serotonin) receptor, subunit 1A (5-HT1AR), gamma-aminobutyric acid type A receptor (GABAAR) and GluN2BNMDAR in the dorsal hippocampal formation (dHF). Furthermore, for the first time, we showed by pharmacological and molecular analysis that the fear memory acquisition is modulated by NMDAR and spontaneous recovery of fear memory, verified after treatment with flavonoidic fractions, may be reliant on tri-activation of GluN2A/ GluN2B-NMDARs and 5-HT1AR in the dHF. ${ }^{8}$ Conversely, our findings also suggested that spontaneous recovery is not modulated by GABAARs. Furthermore, the varying effects of flavones on gene expression may suggest that the dHF is implicated in conditioned suppression, seeming to subserve the neural circuitry involved in conditioned suppression.

\section{Conclusion}

Our results corroborate and advance the knowledge concerning the protective and therapeutic properties of EGb and Flavonoid-rich fraction in short- and long-term memory. The major fear memory/ treatment-dependent changes observed in our study included spontaneous recovery of fear memory. In adult and middle-aged rats this effect may be related to the enhancement of consolidation of fear memory and short-term memory. These findings were further confirmed by data from pharmacological and molecular analysis from dHF obtained from rats treated with $\mathrm{EGb}$ or FfB and from PFC analysis of DNA damage. Together, these findings support and extend the view that flavonoids possess cognitive enhancing properties and provide important information concerning the molecular basis of conditioned suppression and the role of the $\mathrm{DH}$ in this process in addition to the prevention or treatment of neurocognitive impairments.

\section{Acknowledgements}

This study was supported by the São Paulo State Research Foundation (FAPESP) from grant 2009/15229-3 and 2013/20379-9 to SMC. CRZ is a scholar from CAPES. JMC is an investigator of the Brasilian Research Council (CNPq).

\section{Conflicts of interest}

Author declares there are no conflicts of interest.

\section{Funding}

None.

\section{References}

1. Paladini AC, Marder M, Viola H, et al. Flavonoids and the Central Nervous System: from Forgotten Factors to Potent Anxiolytic Compounds. J Pharm Pharmacol. 1999;51(5):519-526.

2. Marder M, Viola H, Wasowski C, et al. 6-Bromoflavone, a high affinity ligand for the central benzodiazepine receptors is a member of a family of active flavonoids. Biochem Biophys Res Commun. 1996;223(2):384 389.

3. Spencer JP, Vauzour D, Rendeiro C. Flavonoids and cognition: The molecular mechanisms underlying their behavioural effects. Arch Biochem Biophys. 2009;492(1-2):1-9.

4. Rendeiro C, Vauzour D, Kean RJ, et al. Blueberry supplementation induces spatial memory improvements and region-specific regulation of hippocampal BDNF mRNA expression in young rats. Psychopharmacology (Berl). 2012;223(3):319-330.

5. Ribeiro ML, Moreira LM, Arçari DP, et al. Protective effects of chronic treatment with a standardized extract of Ginkgo biloba L. in the prefrontal cortex and dorsal hippocampus of middle-aged rats. Behav Brain Res. 2016;313:144-150.

6. Zamberlam CR, Vendrasco NC, Oliveira DR, et al. Effects of standardized Ginkgo biloba extract on the acquisition, retrieval and extinction of conditioned suppression: Evidence that short-term memory and long-term memory are differentially modulated. Physiol Behav. 2016;165:55-68.

7. Oliveira DR, Sanada PF, Saragossa Filho AC, et al. Neuromodulatory property of standardized extract Ginkgo biloba L. (EGb 761) on memory: behavioral and molecular evidence. Brain Res. 2009;1269:68-89.

8. de Oliveira DR, Zamberlam CR, Rêgo GM, et al. Effects of the a flavonoid-rich fraction on the acquisition and extinction of fear memory: Pharmacological and molecular approaches. Front Behav Neurosci. 2016;9:345

9. de Oliveira DR, Zamberlam CR, Gaiardo RB, et al. Flavones from Erythrina falcata are modulators of fear memory. BMC Complement Altern Med. 2014;14:288. 